\title{
Dermatological Disease Classification utilizing 쁬 Image Processing and Neural Networks
}

\author{
Shikha Malik, V.V Dixit
}

\begin{abstract}
Skin diseases are a frequent problem among all age groups. Application of Machine Learning (ML) is exceedingly suitable for skin diseases identification as it has large clinical image database that can be used to train models and interpret diagnosis for better patient outcomes. Researchers have used various image processing techniques and classification methods. Color and Texture based features are most commonly used for image analysis and Convolutional Neural Network (CNN) has become current standard practice in classifying skin disorders. This paper presents a thorough survey of image processing techniques and classifiers for skin diseases detection.
\end{abstract}

Keywords: Image Processing, Machine Learning, Neural Networks

\section{INTRODUCTION}

Skin is the largest sensory organ of our body. Dermatology is the field of medicine concerned with the diagnosis, treatment and prevention of skin disorders. Dermatology deals with morphological features and in majority of cases, diagnosis are based on visual image recognition. Application of Machine Learning (ML) is particularly important due to its's ability for image recognition and classification [1]. A study published in Nature [2] demonstrated that deep learning can classify skin cancer with a level of expertise comparable to dermatologists.

Skin disease detection system based on ML is becoming an important tool for clinical diagnosis of skin diseases. Such system will assist in diagnosis, particularly in enhancing accuracy and sensitivity of identification of skin lesions and rashes, thereby improving patient care [3]. In India, large number of population consults General Practitioners for skin diseases [4]. Such systems could help them to narrow down their differential diagnosis [5]. A typical skin disease recognition system includes series of steps like acquiring the image, pre-processing it, extracting significant features and finally classifying the diseases. This paper presents a comprehensive survey on dermatological disease

Revised Manuscript Received on December 25, 2020.

* Correspondence Author

Shikha Malik*, Research Scholar, G H Raisoni College of Engg \& Management, Pune, Email: malik_shikha.ghrcemetcphd@raisoni.net

Dr. V.V Dixit, Director, RMD Singhad Technical Institutes Campus, Warje, Pune, India. Email: vvdixit73@gmail.com

(c) The Authors. Published by Blue Eyes Intelligence Engineering and Sciences Publication (BEIESP). This is an open access article under the CC BY-NC-ND license (http://creativecommons.org/licenses/by-nc-nd/4.0/) classification systems utilizing image processing and neural networks.

\section{ARCHITECTURE OF SKIN DISEASE DETECTION SYSTEM}

Skin disease recognition system involves four main steps (1) Image sensing and Acquisition (2) Pre-Processing of skin image (3) Skin Feature Extraction (4) Disease classification by suitable classifiers.

(1) Image sensing and Acquisition: Dermoscopic images as well as macroscopic images (clinical images) are extensively used in the ML based techniques for diagnosis of skin diseases. Macroscopic images are captured from smart phone cameras or digital cameras whereas dermoscopic images are acquired from dermatoscope and have the advantage of enhanced visualization of different pigment patterns on skin surface.

(2) Image Pre-Processing: The images acquired from different sources like digital camera or by dermoscopy vary in size and resolution and hence pre-processing is the first step required for producing good quality image. Image pre-processing steps involves standardizing the image by resizing and by removal of unwanted noise like hair, air bubbles and skin pigments with the help of filter. Then by the process of data augmentation like flipping, rotation etc. and segmentation, the image is represented into more meaningful form for easier analysis [8]. Segmentation helps in extracting the region of interest i.e. affected area from background image.

(3) Skin Feature Extraction: This step reduces the dimension of image by removing the redundant information without losing the relevant data. Feature extraction algorithms are used to detect features such as shape, edges or texture in a skin image. There are three commonly used feature extraction algorithms for detection of skin lesions.

- Geometrical Features: These are the features obtained from set of geometric elements like points, lines or curves. Image geometrical features include Asymmetry, Border, Colour and Diameter (ABCD) features.

- Color Features: Color feature extraction is used after the segmentation to identify visible color of image. The various statistical color feature descriptors are mean, variance, standard deviation and skewness.

- Texture Features: Texture is the property of surface which gives important information about the structure of surface.

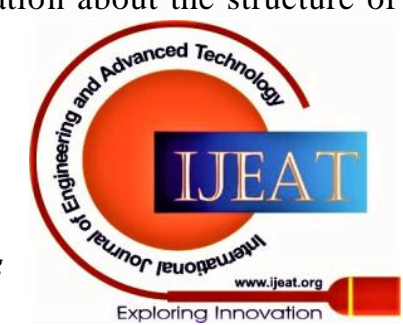




\section{Dermatological Disease Classification utilizing Image Processing and Neural Networks}

- It is a function of spatial variation in pixel intensity in an image [6].

The two widely used texture feature algorithms are local binary pattern operator (LBP) and gray level co-occurrence matrix (GLCM). The various GLCM statistical parameters are Energy, Entropy, Variance, Correlation and Homogeneity.

- All these features together form a feature vector and is used for training the classifier.

(4) Classification: Classifier models are trained on input feature extracted from processed image and then trained models are used for testing new images. This categorises the images into one of the pre-defined diseases class. The commonly used classification algorithms are Linear Discriminative Analysis (LDA), Naive Bayes Classifier (NB), Support Vector Machine (SVM), K-Nearest Neighbour (KNN), Artificial Neural Networks (ANN) and Convolutional Neural Networks (CNN) [7]. Fig. 1 shows the process of skin disease detection.

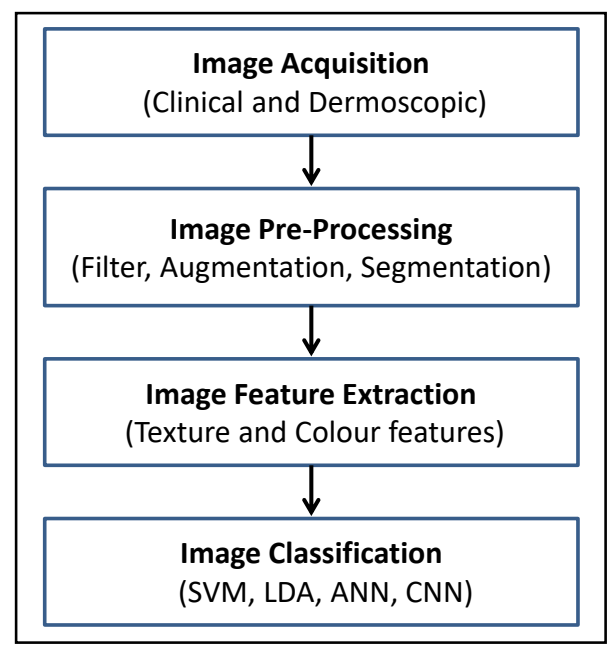

Fig.1. Skin Disease Detection steps

\section{LITERATURE SURVEY ON IMPLEMENTATION OF IMAGE PROCESSING AND NEURAL NETWORK SYSTEMS FOR SKIN DISEASE DETECTION}

Table-I shows summary of work on dermatological skin disease detection using image processing and neural networks.

Rahat Yasir et.al [8] trained neural network with user input features like liquid, age, gender, feeling, elevation etc. and image extracted features like color, shape, area etc. and tested it for 775 skin images of 9 diseases. The proposed model performs better with high elevation features and has low detection rate for low elevation features in affected area.

In [9], authors developed border detection algorithm for identification of non-melanocytic skin lesions (NoMSLs) and melanocytic skin lesions (MSLs). Layer model and flat model are proposed for the classification task and results indicate that layered model perform better than flat model.

Shrivastava VK et al [10] have developed a psoriasis risk assessment system (pRAS) that helps dermatologists in analyzing psoriasis disease at an early stage. Experiments are conducted using several combinations of feature selection methods and classifiers and optimal results are obtained.
Authors of [11] have proposed automatic detection of two misdiagnosed diseases (eczema and psoriasis) by combining both visual and non-visual aspects of diseases. Through K-means clustering segmentation algorithm and by combining categorical and texture features, system achieves accuracy of $84 \%$.

Authors of [12] compared dermatologists and CNN for onychomycosis detection. CNN model trained with clinical images achieved greater accuracy in diagnosing onychomycosis compared to dermatologists. The classifier achieved specificity range of $69.3-96.7 \%$, sensitivity range of 82.7-96.7\%.

Authors of [13] used three deep learning algorithms for automatic skin disease detection. Inception V3, Inception ResNet V2 and MobileNet are used for extracting features. Logistic Regression \& Random Forest classifiers aided in training and testing the model. This system shows $88 \%$ efficiency in classifying 20 diseases accurately. Combining all 3 models has the potential of further improving accuracy.

P R. Hegde et al. [14] extracted GLCM and color features of three diseases and applied LDA, SVM and ANN classifiers. The results proved that LDA and color features is better in two as well as multiclass classification whereas SVM shows better accuracy for multiclass using texture feature and for binary class using hybrid features.

Li-sheng Wei et al. [15] applied water shed algorithm for image segmentation and after extracting GLCM and color features, results are tested with SVM classifier. The proposed method showed improvement in accuracy as compared to other techniques by combining texture and color features.

The authors of [16] worked on the segmentation and automatic classification of Psoriasis skin biopsy images. Super pixel technique is used for segmentation and classification is done by deep CNN model. Results showed CNN with feature based classifiers gives accuracy of $95.17 \%$ and are more efficient than hand-crafted feature based classifiers like SVM, RF and KNN.

In [17] author used pretrained deep CNN for feature extraction and classification of four common cutaneous diseases and tested their performance with the help of experienced dermatologists. Results showed that the average accuracy of evenly distributed dataset is slightly less than uneven dataset. Author also describes four major reasons of misclassification of a disease.

Published By:

Blue Eyes Intelligence Engineering and Sciences Publication

(C) Copyriaht: All rights reserved.

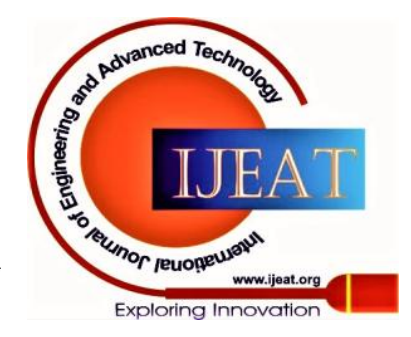


Table-I: Summary of work on dermatological disease detection using Image Processing techniques and Neural Networks

\begin{tabular}{|c|c|c|c|c|c|}
\hline Author \& Year & Disease Type & $\begin{array}{l}\text { Feature extraction } \\
\text { method }\end{array}$ & Classifier & $\begin{array}{l}\text { No of } \\
\text { images } \\
\text { (Type) }\end{array}$ & Evaluation measure \\
\hline $\begin{array}{l}\text { Rahat Yasir, A } \\
\text { Rahman, and Nova } \\
\text { Ahmed } 2014 \text { [8] }\end{array}$ & $\begin{array}{c}\text { Eczema, Acne, } \\
\text { Psoriasis, Vitiligo } \\
\text { Leprosy, , Scabies, } \\
\text { Foot Ulcer, , T } \\
\text { Corporis, P Rosea }\end{array}$ & $\begin{array}{c}\text { Geometrical, color, user input } \\
\text { features (Gender, Age, feeling } \\
\text { etc) }\end{array}$ & ANN & $775(\mathrm{C})$ & $\begin{array}{c}\text { ACC(Psoriasis, Foot Ulcer, } \\
\text { Vitiligo): 90\%, ACC(T } \\
\text { Corporis, P Rosea): } 85 \%\end{array}$ \\
\hline $\begin{array}{l}\text { K. Shimizu et al. } \\
2015 \text { [9] }\end{array}$ & $\begin{array}{l}\text { Melanoma, Nevus, } \\
\text { BCC, SK }\end{array}$ & $\begin{array}{c}\text { Color, Subregion, Texture } \\
\text { Features }\end{array}$ & $\begin{array}{l}\text { Layer model for task } \\
\text { decomposition, Flat } \\
\text { model }\end{array}$ & 968 (D) & $\begin{array}{c}\text { Detection rates-Melanoma: } \\
\text { 90.48\%, Nevi: } 82.51 \% \text {, BCC: } \\
\text { 82.61\%, SK: } 80.61 \%\end{array}$ \\
\hline $\begin{array}{c}\text { Shrivastava } \\
\text { VK, Londhe } \\
\text { ND, Sonawane RS, } \\
\text { and Suri JS } 2017 \\
{[10]} \\
\end{array}$ & Psoriasis & Color, Texture, HOS features & NN , SVM, DT & $670(\mathrm{C})$ & $\begin{array}{l}\text { ACC (FDR+SVM): 99.84\%, } \\
\text { SE: } 99.76 \%, \text { SP: } 99.99 \%\end{array}$ \\
\hline $\begin{array}{c}\text { Uvin Withana, } \\
\text { Pumudu Fernando } \\
2017 \text { [11] } \\
\end{array}$ & Eczema, Psoriasis & $\begin{array}{l}\text { Texture, Area-To-Perimeter } \\
\text { Ratio, Categorical variables }\end{array}$ & SVM & $100(\mathrm{C})$ & ACC: $100 \%$ \\
\hline $\begin{array}{c}\text { Han SS et al. } 2018 \\
{[12]}\end{array}$ & Onychomycosis & CNN (convolutional layer) & $\mathrm{CNN}$ & 49,567 (C) & $\begin{array}{l}\text { SP: } 69.3-96.7 \% \text {, } \\
\text { SE: 82.7-96.7\%. }\end{array}$ \\
\hline $\begin{array}{c}\text { Sourav Kumar } \\
\text { Patnaik et al. } 2018 \\
{[13]} \\
\end{array}$ & 20 diseases & $\begin{array}{l}\text { CNN (3 DL architecture: } \\
\text { mobilenet, Inception V2, } \\
\text { Inception V3) }\end{array}$ & $\begin{array}{l}\text { Logistic Regression } \\
\text { or Random Forest }\end{array}$ & $1000(\mathrm{C})$ & Better ACC (Inception V3): 88\% \\
\hline $\begin{array}{l}\text { P R. Hedge,M M. } \\
\text { Shenoy and B.H. } \\
\text { Shekar } 2018 \text { [14] }\end{array}$ & $\begin{array}{l}\text { Eczema, Lichen } \\
\text { planus, Psoriasis }\end{array}$ & $\begin{array}{c}\text { Color, GLCM Texture } \\
\text { feature, Segmentation - HED }\end{array}$ & SVM, LDA, ANN & $310(\mathrm{C})$ & $\begin{array}{l}\text { ACC (Texture Feature +SVM ): } \\
80 \% \text {, ACC (Color Feature + } \\
\text { LDA ): } 82.58 \%\end{array}$ \\
\hline $\begin{array}{c}\text { Li-sheng Wei,Quan } \\
\text { Gan and Tao Ji } 2018 \\
{[15]} \\
\end{array}$ & $\begin{array}{l}\text { Herpes, Dermatitis, } \\
\text { Psoriasis }\end{array}$ & $\begin{array}{l}\text { GLCM Texture, Color } \\
\text { Feature, Vertical Image } \\
\text { Segmentation } \\
\end{array}$ & SVM & $90 \quad(\mathrm{C})$ & ACC: $90 \%$ \\
\hline $\begin{array}{l}\text { Anabik Pal et al. } \\
2018 \text { [16] }\end{array}$ & Psoriasis & $\begin{array}{l}\text { Superpixel segmentation- } \\
\text { SLIC }\end{array}$ & $\begin{array}{c}\text { U shaped fully } \\
\text { convolutional neural } \\
\text { network(FCN) } \\
\end{array}$ & $90(\mathrm{C})$ & ACC: $95.17 \%$ \\
\hline $\begin{array}{l}\text { Xinyuan Zhang et } \\
\text { al.2018 [17] }\end{array}$ & $\begin{array}{l}\text { Melanocytic nevus, } \\
\text { SK, BCC, Psoriasis }\end{array}$ & $\begin{array}{l}\text { Pooling, convoutional layers } \\
\text { of Inception v3 model }\end{array}$ & DCNN & $\begin{array}{l}\text { Dataset A: } \\
\text { 1067, } \\
\text { Dataset B: } \\
\text { 528(D) }\end{array}$ & $\begin{array}{l}\text { ACC(Dataset A): 87.25\% } \\
\text { ACC(Dataset B): } 86.6 \%\end{array}$ \\
\hline $\begin{array}{c}\text { Mohammed } \\
\text { A.Al-masni et al. } \\
2018[18] \\
\end{array}$ & Melanoma, SK & CNN (convolutional layer) & $\mathrm{FrCN}$ & 2750 (D) & $\begin{array}{c}\text { Jaccard index - 77.11\%, ACC } \\
\text { (Melanoma): } 90.78 \% \\
\text { ACC(SK): } 91.29 \% \\
\end{array}$ \\
\hline $\begin{array}{c}\text { A Rezvantalab, H } \\
\text { Safigholi, S } \\
\text { Karimijeshni } 2018 \\
{[19]} \\
\end{array}$ & 10 diseases & Pretrained CNN & $\begin{array}{c}\text { DenseNet 201, } \\
\text { ResNet 152, } \\
\text { Inception v3, } \\
\text { Inception ResNet v2 } \\
\end{array}$ & 10135 (D) & $\begin{array}{c}\text { ROC AUC (ResNet } \\
\text { 152)-94.40\% , (DenseNet } 201 \text { ) } \\
-99.30 \%\end{array}$ \\
\hline $\begin{array}{c}\text { Jordan Yap, } \\
\text { William Yolland } \\
\text { and Philipp Tschand } \\
2018 \text { [20] } \\
\end{array}$ & $\begin{array}{c}\text { Naevus, Melanoma, } \\
\text { BCC, SCC, } \\
\text { Pigmented Benign } \\
\text { Keratosis } \\
\end{array}$ & CNN (Pooling layers) & 2 ResNet-50 & 2917 (C,D) & AUC:86.6\% \\
\hline $\begin{array}{l}\text { R. B. Oliveira . et al. } \\
2019 \text { [21] }\end{array}$ & Melanoma & $\begin{array}{c}\text { Hybrid-Shape+Color+Textur } \\
\text { e features } \\
\text { Feature Selection: GRFS, } \\
\text { CFS } \\
\end{array}$ & $\begin{array}{l}\text { KNN, Bayes net, } \\
\text { MLP, OPF }\end{array}$ & $1104 \quad$ (C) & $\begin{array}{l}\text { ACC( GRFS+MLP ): } 90.6 \% \\
\text { ACC ( CFS + OPF ): } 91.6 \%\end{array}$ \\
\hline $\begin{array}{l}\text { Nawal Soliman } \\
2019 \text { [22] }\end{array}$ & $\begin{array}{l}\text { Eczema, Melanoma, } \\
\text { Psoriasis }\end{array}$ & Pre-trained CNN & SVM & $80(\mathrm{C})$ & ACC: $100 \%$ \\
\hline $\begin{array}{l}\text { T. Y. Tan, L. Zhang } \\
\text { and C. P. Lim } 2019 \\
{[23]} \\
\end{array}$ & $\begin{array}{l}\text { Actinic Keratosis, } \\
\text { Nevus, Melanoma }\end{array}$ & $\begin{array}{c}\text { ABCD ,Texture Feature. } \\
\text { Feature Selection - APSO, } \\
\text { RCPSO } \\
\end{array}$ & $\begin{array}{c}\text { Ensemble model } \\
\text { (KNN ,SVM, Deep } \\
\text { CNN) } \\
\end{array}$ & 1300 (D) & $\begin{array}{l}\text { High performance, Low } \\
\text { computational cost }\end{array}$ \\
\hline $\begin{array}{c}\text { Muhammad Qasim } \\
\text { Khan et al. } 2019 \\
\text { [24] }\end{array}$ & Melanoma, Nevus & $\begin{array}{l}\text { Hybrid - GLCM+LBP + color } \\
\text { features }\end{array}$ & SVM & 397 (D) & $\begin{array}{c}\text { ACC (Color feature + SVM ): } \\
77 \% \\
\text { ACC (Hybrid Feature + SVM): } \\
96 \% \\
\end{array}$ \\
\hline $\begin{array}{l}\text { Jian Liu et al. } 2019 \\
\text { [25] }\end{array}$ & Vitiligo & $\mathrm{CNN}$ & $\begin{array}{l}\text { pretrained CNN } \\
\text { models: } \\
\text { Vgg16,Xception, } \\
\text { Inceptionv3 } \\
\end{array}$ & 38677 (D) & ACC(Average): 87.8\% \\
\hline $\begin{array}{l}\text { Ziying Vanessa Lim } \\
\text { et al. } 2019 \text { [26] }\end{array}$ & Acne Vulgaris & CNN (Convolutional layer) & $\begin{array}{c}\text { 3 CNN Architectures: } \\
\text { Dense Net,Inception } \\
\text { v4,ResNet } 18 \\
\end{array}$ & $472(\mathrm{C})$ & $\begin{array}{c}\text { Better ACC (Inception V4): } \\
67 \% \text {, Pearson Correlation: } 0.77\end{array}$ \\
\hline $\begin{array}{l}\text { BelaL Ahmad et al. } \\
2020 \text { [27] }\end{array}$ & $\begin{array}{c}\text { Acne, Spots, } \\
\text { Blackheads, Dark } \\
\text { circles } \\
\end{array}$ & $\begin{array}{l}\text { Triplet loss functions for } \\
\text { discriminative features }\end{array}$ & $\begin{array}{c}\text { Deep CNN models, } \\
\text { ResNet 152, } \\
\text { Inception ResNet V2 }\end{array}$ & $14000(\mathrm{C})$ & Better ACC , SP \\
\hline
\end{tabular}




\section{Dermatological Disease Classification utilizing Image Processing and Neural Networks}

CNN convolutional neural network, SK seborrheic keratosis, SCC squamous cell carcinoma, FrCN full resolution convolutional network, $K N N$ k- nearest neighbor, $S V M$ support vector machine, $L D A$ linear discriminative analysis, $A N N$ artificial neural network, $D C N N$ deep convolutional neural network, $B C C$ basal cell carcinoma, AUROC area under receiver operating characteristics, LR logistic regression, $R F$ random forest, DT Decision tree ACC accuracy, SP specificity, SE sensitivity, OPF optimum path classifier, C clinical, $D$ dermoscopic

In [18] author proposed automatic segmentation method using Full resolution convolutional networks. This technique extracts full resolution features and does not require any other processing. Results exhibit better performance than Fully Convolutional Network (FCN), SegNet and U-Net deep learning algorithms.

Authors of [19] tested performance of three different pre-trained CNN architectures on 10135 dermoscopic images and classified 8 categories of skin diseases. Results proved that all these models are $11 \%$ more accurate than highly skilled dermatologists.

In [20] authors have done combined analysis of clinical, dermoscopic images and patient metadata demonstrated that the multi modal classifier outperforms single classifier in distinguishing multiple skin diseases.

Roberta B. et al [21] used various combinations of features like texture, shape and color features for feature extraction of skin cancer images. Several feature selection methods like gain ratio-based feature selection (GRBFS), correlation based feature selection (CFS) are used along with different classifiers and their performance are compared for obtaining better results. Results showed Optimum Path Forest (OPF) classifier gives accuracy of $92.3 \%$ without feature selection and $91.6 \%$ accurate with correlation feature selection Algorithm.

In [22], authors proposed image processing based system for the detection of three common skin diseases (eczema, melanoma, psoriasis). It includes pre-processing by resizing the image and feature extraction using pretrained five layer AlexNet-CNN model. SVM classifier is used for four class classification. The system achieves $100 \%$ accuracy in detecting all three diseases.

In the work presented in [23], first texture features are extracted using local binary patterns, gray length run length matrix, and histogram oriented gradient operators. Then these in combination with ABCD features are optimized using two particle swarm optimization algorithms. Finally combination of KNN, SVM and Deep CNN models are used for classifying skin diseases. All experiments are done on medical dataset from UCI - ML repository and Dermoscopic skin lesion images.

In [24] author proposed a system consisting of hybrid feature extraction techniques like combination of color, GLCM and LBP features which results in significant improvement in accuracy. Centroid selection approach for $\mathrm{K}$ means clustering algorithm for segmenting the images is effective as compared to standard method. Statistical results showed that hybrid feature method provides better results than individual feature extraction methods.

Authors of [25] proposed detection of Vitiligo using average results of three pretrained CNN models trained on RGB, HSV and YCrCb color space images. The applied strategy provides classification accuracy of $87.8 \%$ and performs better than individual networks.

In [26] author tested 3 CNN architectures for 3 classes of acne severity: clear, mild and severe. Inception V4 achieves

highest accuracy of $67 \%$ on larger image size proving deep learning is efficient for large data set and high resolution images. Approached discussed in [27] uses Triplet loss function along with deep CNN ResNet152 and Inception ResNet-V2 models for achieving better accuracy in effective classification of skin diseases. In Triplet loss function method L2 distance is calculated among images in Euclidean space and on the basis of distance, images are classified into 4 categories as acne, dark circles, blackheads and spots.

\section{CONCLUSION}

This survey focuses on image processing techniques and neural networks employed for the detection of various dermatological disorders.

Recent trends and issues related to detection of various dermatological disorders using image processing and neural networks can be summarized as:

Majority of the research work has been done on detection of skin cancer compared to that of other common skin diseases such as psoriasis, eczema, acne, vitiligo etc. Within skin cancer, majority of research has been done on Melanocytic Skin Lesion whereas non-melanocytic skin lesions are relatively neglected.

Dermoscopic and clinical images have been extensively used for diagnosis however dermoscopic images provide better visualization and fine details.

Combinations of color and texture features are considered more frequently for feature extraction in majority of studies.

Various classification methods including SVM, LDA, KNN etc. have been utilized for skin disease classification, however, End to End trained CNNs have become current standard practice. Ensemble methods combining different classifiers have shown improved predictive performance of detection system.

In diagnosis of skin diseases, dermatologists usually identify based on other information (patient history, age, demographics etc.) than only images. This information should be embedded in algorithm to get better accuracy in diagnosis.

\section{REFERENCES}

1. Li C, Shen C, Xue K, Shen X, Jing Y, Wang Z et al., “ Artificial intelligence in dermatology, Chinese Medical Journal, Volume 132, Issue 17,pp. 2017-2020 , 2019.

2. Esteva, A., Kuprel, B., Novoa, R.A., "Dermatologist-level classification of skin cancer with deep neural networks". Nature 542, pp.115-118,2017.

3. Adamson A, Smith A. "Machine Learning and Health Care Disparities in Dermatology, JAMA Dermatology vol. 154, Issue 11, pp. 1247-1248,2018.

4. J. Rathod, V. Waghmode, A. Sodha and P. Bhavathankar, "Diagnosis of skin diseases using Convolutional Neural Networks," Second International Conference on Electronics, Communication and Aerospace Technology (ICECA), Coimbatore, 2018 ,pp. 1048-1051, 2018, doi: 10.1109/ICECA.2018.8474593.

Published By:

Blue Eyes Intelligence Engineering and Sciences Publication

(C) Copyright: All rights reserved.

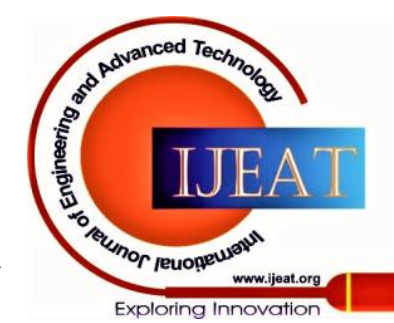


5. Du-Harpur X, Watt FM, Luscombe NM, Lynch MD. What is AI? Applications of artificial intelligence to dermatology [published online ahead of print, 2020 Jan 20]. Br J Dermatol.,2020

6. R. M. Haralick, K. Shanmugam and I. Dinstein, "Textural Features for Image Classification," in IEEE Transactions on Systems, Man, and Cybernetics, vol. SMC-3, no. 6, pp. 610-621, Nov. 1973.

7. Oliveira R.B., Papa J.P., Pereira A.S. et al., " Computational methods for pigmented skin lesion classification in images: review and future trends." Neural Comput \&Applic 29, pp. 613-636, 2018

8. R. Yasir, M. A. Rahman and N. Ahmed, "Dermatological disease detection using image processing and artificial neural network," 8th International Conference on Electrical and Computer Engineering, Dhaka, 2014, pp. 687-690,2014

9. K. Shimizu, H. Iyatomi, M. E. Celebi, K. Norton and M. Tanaka, "Four-Class Classification of Skin Lesions With Task Decomposition Strategy," in IEEE Transactions on Biomedical Engineering, vol. 62, no. 1, pp. 274-283, Jan. 2015

10. V.K. Shrivastava, N.D. Londhe, R.S. Sonawane, J.S. Suri, "A novel and robust Bayesian approach for segmentation of psoriasis lesions and its risk stratification", Comput. Methods Programs Biomed., vol. 150, pp. 9-22, Oct. 2017.

11. U. Withana and P. Fernando, "Differential diagnosis of eczema and psoriasis using categorical data in image processing," 2017 Seventeenth International Conference on Advances in ICT for Emerging Regions (ICTer), Colombo, 2017, pp. 1-6, 2017.

12. Han SS, Park GH, Lim W, Kim MS, Na JI, Park I, et al., "Deep neural networks show an equivalent and often superior performance to dermatologists in onychomycosis diagnosis: Automatic construction of onychomycosis datasets by region-based convolutional deep neural network". PLoS ONE 13, pp. 1-14, 2018

13. Patnaik S. K, Sidhu M. S, Gehlot Y, Sharma B, Muthu P., "Automated Skin Disease Identification using Deep Learning Algorithm." Biomedical and Pharmacology Journal, vol. 11, no. 3, 2018

14. Hegde P.R., Shenoy M.M., Shekar B.H., "Comparison of Machine Learning Algorithms for Skin Disease Classification Using Color and Texture Features," 2018 International Conference on Advances in Computing, Communications and Informatics (ICACCI), Bangalore, 2018, pp. 1825-1828, 2018

15. L. Wei, Q. Gan, T. Ji, "Skin Disease Recognition Method Based on Image Color and Texture Features", Computational and Mathematical Methods in Medicine, vol. 2018, pp. 2-3, 2018.

16. Pal A, Garain U, Chandra A, Chatterjee R, Senapati S., "Psoriasis skin biopsy image segmentation using Deep Convolutional Neural Network", Computer methods and programs in biomedicine, vol. 159, pp.59-69, 2018

17. X. Zhang, S. Wang, J. Liu, C. Tao, "Towards improving diagnosis of skin diseases by combining deep neural network and human knowledge", BMC medical informatics and decision making,vol. 18,Suppl 2 ,pp.59, 2018

18. M. A. Al-masni, M. A. Al-antari, M.-T. Choi, S.-M. Han, T.- S. Kim, "Skin lesion segmentation in dermoscopy images via deep full resolution convolutional networks", Computer Methods and Programs in Biomedicine ,vol. 162 ,pp. $221 \quad-231,2018$. doi:https://doi.org/10.1016/j.cmpb.2018.05.027.

19. A. Rezvantalab, H. Safigholi, S. Karimijeshni, " Dermatologist level dermoscopy skin cancer classification using different deep learning convolutional neural networks algorithms", arXiv:1810.10348,2018

20. J. Yap, W. Yolland, P. Tschandl, "Multimodal skin lesion classification using deep learning", Exp. Dermatol., vol. 27, pp. 1261-1267, 2018

21. R. B. Oliveira, A. S. Pereira, J. M. R. S. Tavares, "Computational diagnosis of skin lesions from dermoscopic images using combined features", Neural Computing and Applications 31, pp. 6091-6111, 2019

22. ALKolifi ALEnezi N, "A Method Of Skin Disease Detection Using Image Processing And Machine Learning". Procedia Computer Science, 163 pp.85-92, 2019.

23. T.Y. Tan, L. Zhang, C.P. Lim, "Intelligent skin cancer diagnosis using improved particle swarm optimization and deep learning models", Applied Soft Computing, vol. 84, 2019.

24. M. Q. Khan et al., "Classification of Melanoma and Nevus in Digital Images for Diagnosis of Skin Cancer", IEEE Access, vol. 7, pp. 90132-144, 2019.

25. Jian Liu, Jianwei Yan, Jie Chen, Guozhong Sun, and Wei Luo, "Classification of vitiligo based on convolutional neural network," International Conference on Artificial Intelligence and Security, ICAIS 2019, Springer, vol 11633 , pp. 214-223, 2019.

26. Lim Z, Akram F, Ngo C, Winarto A, Lee W, Liang K et al. ,“Automated grading of acne vulgaris by deep learning with convolutional neural

Published By:

Blue Eyes Intelligence Engineering and Sciences Publication (C) Copyright: All rights reserved. networks". Skin Research and Technology.,vol. 26(2):pp.187-192,2019.

27. B. Ahmad, M. Usama, C. Huang, K. Hwang, M. S. Hossain and G. Muhammad, "Discriminative Feature Learning for Skin Disease Classification Using Deep Convolutional Neural Network," IEEE Access, vol. 8, pp. 39025-39033, 2020, doi: 10.1109/ACCESS.2020.2975198.

\section{AUTHORS PROFILE}

Ms Shikha Malik awarded MTech. degree in ECE from Kurukshetra University in 2010 and currently pursuing Ph.D from GH Raisoni college of Engineering and Management , Pune. She is presently working as an Assistant Professor in Atharva College of Engineering, Mumbai. She has 12 yrs of teaching experience. Her research areas include Bio medical image processing and Machine Learning.

Dr. Vaibhav Vitthalrao Dixit received his Ph.D degree in E\&TC Engineering in 2015 from SPPU, Pune, (India). Presently he is working as Principal, RMD Singhad School of Engineering and Director of RMD Singhad Technical Institutes Campus, Warje, Pune, (India). His research areas include Pattern recognition, neural networks and Bio medical applications. He has 19 yrs of teaching and 5 yrs industrial experience 